\title{
Multi-model ensemble: technique and validation
}

\author{
J. R. Rozante, D. S. Moreira, R. C. M. Godoy, and A. A. Fernandes \\ Center for Weather Forecasting and Climate Studies, INPE, Cachoeira Paulista, SP, Brazil \\ Correspondence to: J. R. Rozante (roberto.rozante@cptec.inpe.br) \\ Received: 17 March 2014 - Published in Geosci. Model Dev. Discuss.: 6 May 2014 \\ Revised: 28 August 2014 - Accepted: 11 September 2014 - Published: 14 October 2014
}

\begin{abstract}
In this study, a method of numerical weather prediction by ensemble for the South American region is proposed. This method takes into account combinations of the numerical predictions of various models, assigning greater weight to models that exhibit the best performance. Nine operational numerical models were used to perform this study. The main objective of the study is to obtain a weather forecasting product (short-to-medium range) that combines what is best in each of the nine models used in the study, thus producing more reliable predictions. The proposed method was evaluated during austral summer (December 2012, and January and February 2013) and winter (June, July and August 2013). The results show that the proposed method can significantly improve the results provided by the numerical models and consequently has promising potential for operational applications in any weather forecasting center.
\end{abstract}

\section{Introduction}

Numerical weather prediction models are important tools for the understanding of meteorological phenomena, as well as for making weather forecasts. The predictability provided by these numerical models shows a strong dependence on initial conditions provided to the model and has been widely discussed since the 1960s (Lorenz, 1965, 1969) to the current decade (Ngan and Eperon, 2012; Cintineo and Stensrud, 2013). Errors that occur during the specification of initial conditions may cause large uncertainties in numerical prediction systems (Thompson, 1957; Zhu and Thorpe, 2006). Other sources of uncertainty may be associated with the representation of physical processes in the models (Krishnamurti et al., 2004; van Lier et al., 2012). In this sense, knowledge of systematic errors occasioned by these uncertainties is of paramount importance in the realization of improvements in the forecasting system, with a view to minimizing the errors, and helping meteorologists in the preparation of weather forecasts.

The technique of combining forecasts made by numerical models has been well explored by various researchers (Tebaldi et al., 2004; Weigel et al., 2010; Chandler et al., 2013). Almost all of the articles that have been written on this subject agree that the combination of several different forecasts provides significant improvements. The questions that arise are those dealing with the method used to combine the forecasts. Recent research in climate modeling suggests that combination schemes with unweighted means provide better results than schemes with weighting based on the performance of each model (Christensen et al., 2010; Déqué and Somot, 2010). And, according to Weigel et al. (2010) and Knutti et al. (2010), the combination of models taking into account the concept of weighting must be treated with great care, principally when applied to climate change.

Now in the area of weather forecasting, intercomparisons among forecasts from different types of numerical models have shown that the performance of each can vary in time as well as in space (Saulo et al., 2001; Silva Dias et al., 2006). Thus, a combination among the results of various types of models, considering the performance of each model, can produce forecasts of greater reliability (Johnson and Swinbank, 2009; Roy Bhowmik and Durai, 2010; Kotal and Roy Bhowmik, 2011). The concept of using the combination of a set of numerical results for the improvement of the prediction models was first discussed by Krishnamurti et al. (1999, 2000a, b) and has been widely used (Yun et al., 2003; Chakraborty et al., 2007; Lenartz et al., 2010).

Roy Bhowmik and Durai (2008, 2010), applying a linear regression technique to a set of forecasts, made by four numerical models, showed that combining the predictions of each model and their respective correlation coefficients 
Table 1. Principal characteristics of the models utilized.

\begin{tabular}{llll}
\hline Abbreviation & $\begin{array}{l}\text { Model } \\
\text { description }\end{array}$ & $\begin{array}{l}\text { Horizontal } \\
\text { resolution }\end{array}$ & $\begin{array}{l}\text { Vertical } \\
\text { levels }\end{array}$ \\
\hline T062ACO & Global - coupled atmosphere-ocean & $\sim 200 \mathrm{~km}$ & 28 \\
T126 & Global - control member of ensemble & $\sim 100 \mathrm{~km}$ & 28 \\
T126MED & Global - mean member of ensemble & $\sim 100 \mathrm{~km}$ & 28 \\
T213 & Global & $\sim 63 \mathrm{~km}$ & 42 \\
T299 & Global & $\sim 44 \mathrm{~km}$ & 64 \\
Eta40 & Regional - control member & $40 \mathrm{~km}$ & 38 \\
Eta40ZHAO & Regional - physics member & $40 \mathrm{~km}$ & 38 \\
Eta15 & Regional & $15 \mathrm{~km}$ & 50 \\
BRAMS20 & Regional & $20 \mathrm{~km}$ & 38 \\
\hline
\end{tabular}

produces significant improvements in predictions of precipitation over India. In other recent studies, addressing the monsoons of India, Krishnamurti et al. (2009), Mitra et al. (2011) and Kumar et al. (2012) assert that - when predictions are generated from a set of numerical models - their quality is improved and their mean square errors reduced.

Currently, the Center for Weather Forecasting and Climate Studies, National Institute for Space Research (CPTEC/INPE) operationally incorporates different modalities of numerical models (general circulation, oceanicatmospheric, regional and atmospheric chemistry). In order to make use in the best possible way of all of the forecasts integrated within CPTEC/INPE models. This paper proposes the development of a product for short- and medium-range weather prediction, which will henceforth be called the Super Model Ensemble of CPTEC (SMEC). The objective of this product is to weight the prediction of each model by the inverse of its own systematic error, so that models with greater error will have less weight and vice versa, thus producing more reliable predictions.

\section{Methodology}

\subsection{Data set}

In the present paper, SMEC will be constructed from four configurations of the CPTEC/INPE's general circulation model (spectral with triangular truncation and Gaussian grid), three configurations of the Eta regional model and one Brazilian-developed version of the regional Atmospheric Modeling System (BRAMS). All models use as initial conditions the analyses produced at the National Centers for Environmental Prediction (NCEP), and they are integrated at different spatial resolutions. A bilinear interpolation is applied to all models in order to standardize them at a resolution of $20 \mathrm{~km}$ (the same as that of the SMEC). More details on the models and their configurations can be seen in Table 1. The period of integration and frequency of outputs used in all models were 7 days and $6 \mathrm{~h}$, respectively.
The SMEC product will be generated over an area including South America and adjacent oceans $\left(90-20^{\circ} \mathrm{W}, 55^{\circ} \mathrm{S}-\right.$ $15^{\circ} \mathrm{N}$ ). This restriction is due to the use of regional models that provide forecasts covering only this domain. We chose to generate the SMEC at a horizontal resolution of $20 \mathrm{~km}$, a resolution that enables detection of mesoscale phenomena and that has the same grid as the precipitation product from MERGE (Rozante et al., 2010), which will be used for validation of SMEC. For the vertical resolution five levels in pressure coordinates $(1000,925,850,500$ and $250 \mathrm{hPa})$ will be used. These levels were selected in order to represent the atmosphere at low, medium and high levels, and also because these are the levels most commonly analyzed by meteorologists in preparing weather forecasts. The Grid Analysis and Display System (GrADS) was used to calculate the weights (errors of the models), to obtain SMEC and all figures presented in this document. The GrADS code package can be obtained from the Operational Implementation group (IO) at CPTEC/INPE (io@cptec.inpe.br).

The variables available in SMEC are air temperature, zonal and meridional wind, geopotential height, relative humidity, vertical velocity at all pressure levels, plus air temperature at $2 \mathrm{~m}$, zonal and meridional wind at $10 \mathrm{~m}$, pressure reduced to mean sea level and precipitation. These are the variables most used in forecasts and are the basis of many other meteorological variables. The length of the SMEC forecast will be 7 days with outputs every $6 \mathrm{~h}$. The product will be evaluated for the months of an austral summer (December 2012, January and February 2013) and an austral winter (June, July and August 2013) and its operational forecasts will be made available in a public area, thus enabling the use of SMEC in the generation of meteorological reports by any weather center.

\subsection{Calculation of the errors of the SMEC}

The bias or mean error $(\sigma)$ of the numerical models is expressed by the difference between the forecasts and the 


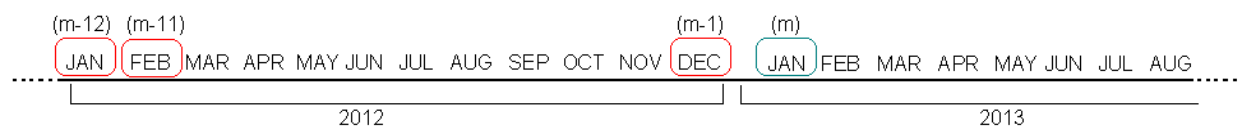

Figure 1. Diagram showing which of the months of forecasts (highlighted in red) will be used for the calculation of the mean bias for the month of January 2013 (highlighted in green).

observations/analyses, given by the following equation:

$\sigma_{(M, v, x, y, z, t)}=\frac{1}{N} \sum_{d=1}^{d=N}\left(F_{(M, v, x, y, z, t)}-A_{(v, x, y, z, t)}\right)$,

where $F, A$ and $N$ indicate forecasts, analyses and number of days of forecasts, respectively. The errors are calculated as a function of model $(M)$, variable $(v)$, longitude $(x)$, latitude $(y)$, vertical level $(z)$ and time of integration $(t)$. This difference indicates tendencies of the forecasts to underestimate or overestimate the values of the meteorological variables. For the case of the precipitation variable, the mean error is obtained utilizing the MERGE product as a substitute in the analyses $(A)$.

The bias of the models varies throughout the year by virtue of the change of seasons. Taking this fact into account, it was decided to calculate the mean bias considering integrations of $168 \mathrm{~h}$ (in $6 \mathrm{~h}$ intervals) throughout a period of 3 months. The determination of the mean bias $\left(\overline{\sigma_{(\mathrm{m})}}\right)$ that will be used in a given month $(\mathrm{m})$, for each model and time of integration, is given by Eq. (2):

$\overline{\sigma(m)}=\frac{1}{D} \sum_{d=1}^{d=D}\left(F_{d}-A_{d}\right)$

where $D$ is the total number of days in the 3 months used in the calculation and $n D$ is the number of days in each month used, expressed in Eq. (3) as

$D=n D_{(m-1)}+n D_{(m-12)}+n D_{(m-11)}$.

The diagram below (Fig. 1) illustrates the period used in the calculation of the mean bias to be used for the SMEC forecasts in the month of January of 2013.

$$
\begin{aligned}
D & =n D_{(\mathrm{DEC})}+n D_{(\mathrm{JAN})}+n D_{(\mathrm{FEB})} \\
D & =31+31+29=91 \\
\sigma_{(\mathrm{JAN})} & =\frac{1}{91} \sum_{d=1}^{d=91}\left(F_{d}-A_{d}\right)
\end{aligned}
$$

For the calculation of the mean square error $\left(\overline{\sigma_{(\mathrm{m})}^{2}}\right)$ the same methodology as described above was applied, given by the following equation:

$\overline{\sigma_{(m)}^{2}}=\frac{1}{D} \sum_{d=1}^{d=D}\left(F_{d}-A_{d}\right)^{2}$.
The SMEC, in general terms, is a product obtained from combinations of numerical predictions of various models. In this case, the bias of each model is removed, and then an average weighted by the reciprocal of the mean square error of each model is calculated. This procedure minimizes the systematic errors of the models, and in addition it assigns greater weight to models that have the lowest values of mean square error. The SMEC can be expressed by the following equation:

$$
\operatorname{SMEC}(v, x, y, z, t)=\frac{\sum_{i=1}^{i=M}\left(\frac{F_{i}(v, x, y, z, t)-\overline{\sigma_{i}}(v, x, y, z, t)}{\overline{\sigma_{i}^{2}}(v, x, y, z, t)}\right)}{\sum_{i=1}^{i=M}\left(\frac{1}{\overline{\sigma_{i}^{2}}(v, x, y, z, t)}\right)} .
$$

\section{Results}

In this section, the results in terms of the contribution of each model to the calculation of the SMEC will be presented, along with statistics of the performance of the product, both for the summer and winter trimesters. The contributions will be shown in terms of the root mean square error (RMSE $=\sqrt{\overline{\sigma^{2}}}$ ), while the performance of the SMEC will be evaluated using the observed data and NCEP analyses.

\subsection{Contribution of the models}

Examples of the contribution of each model to the calculation of the SMEC will be given for some variables, levels and times of integration. The identification of the regions in which each model has its greatest contribution was performed as follows: at each grid point over the South American domain, a numeric value has been assigned for the model having the lowest value of RMSE of all. This characterizes a spatial distribution of the regions where each model has its greatest contribution to the calculation of the SMEC.

Figure 2 shows the spatial distribution of grid points at which each model has the lowest RMSE values and, consequently, the greatest contribution to the calculation of the SMEC. The numbers inside the captions indicate the percentage of grid points at which the model obtained the lowest values of RMSE. The right column shows the results for the summer period; those for winter are on the left.

Figure 2 shows the spatial distribution of the grid points with the lowest values of RMSE for all models. In the case of the $48 \mathrm{~h}$ integrations for mean sea level pressure in winter (Fig. 2a), it appears that the Eta regional models (Eta15, 

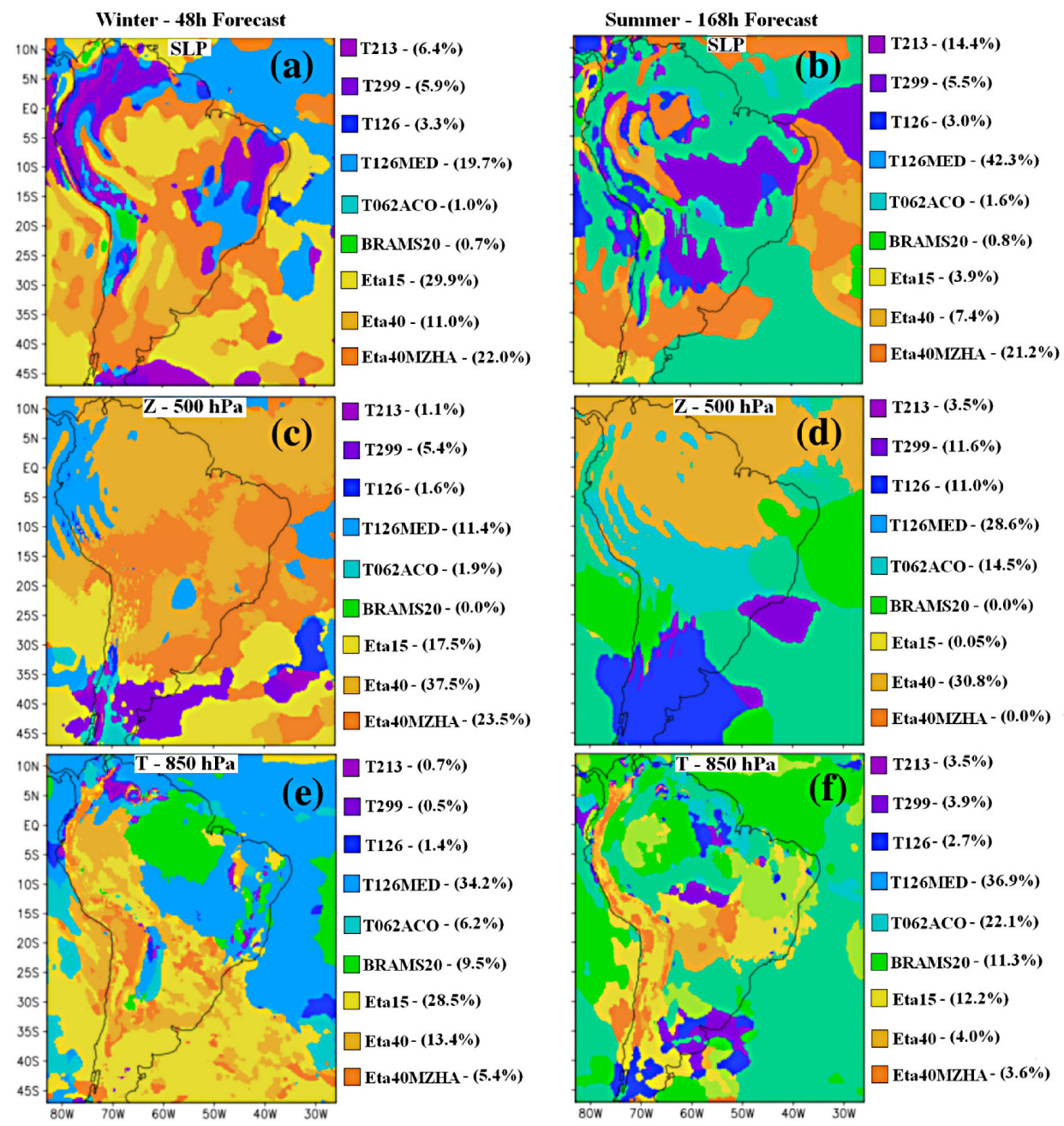

Figure 2. Spatial distribution of grid points from models with smallest values for RMSE of mean sea level pressure (a, b), $500 \mathrm{hPa}$ height (c, d) and $850 \mathrm{hPa}$ air temperature (e, f), for winter (left column) and summer (right column).

Eta40 and Eta40ZHAO) have smaller errors in comparison to the other models, Eta15 being responsible for almost $30 \%$ of these grid points in the domain. The major contribution from global models is attributed to the T126MED model, totaling almost $20 \%$ of the points. The results for $168 \mathrm{~h}$ of integration (Fig. 2b), during the winter, indicate that the global models show more areas of errors that are smaller than the errors in the regional models, with the T126MED being responsible for over $42 \%$ of the total area. For $48 \mathrm{~h}$ forecasts of $500 \mathrm{hPa}$ geopotential height (Fig. 2c) during the winter, the three Eta models predominate, totaling around $78 \%$ of the field, with the Eta40 responsible for more than $37 \%$. However, for $168 \mathrm{~h}$ forecasts in summer (Fig. 2d), the global models stood out, accounting for more than half (around $69 \%$ ) of the domain having the lower values of RMSE. In this case among the global models, the T126ME covered the largest percentage of area (more than $28 \%$ ). The remaining fraction was almost entirely occupied by the Eta40 forecasts (more than 30\%).
For the case of $48 \mathrm{~h}$ forecasts of the $850 \mathrm{hPa}$ temperature during winter (Fig. 2e), the regional models predominate in the southwestern part of the domain and the global models, mainly the T126MED, in the northeastern part. At $168 \mathrm{~h}$ in winter (Fig. 2f), the most striking feature was the predominance on the part of the global models over the Atlantic and Pacific oceans, primarily by the T126MED which reached almost $37 \%$.

Figure 3 shows the time evolution of the mean (over the entire domain) of the percentage of grid points where the models showed the lowest values of RMSE. In this case, these points were computed only in terms of global and regional models and a combined winter-summer average. For the annual mean of sea level pressure (Fig. 3a), as well as for $500 \mathrm{hPa}$ geopotential height (Fig. 3b) (and other variables not shown), it is found that regional models are responsible for the largest number of grid points with smallest values of 

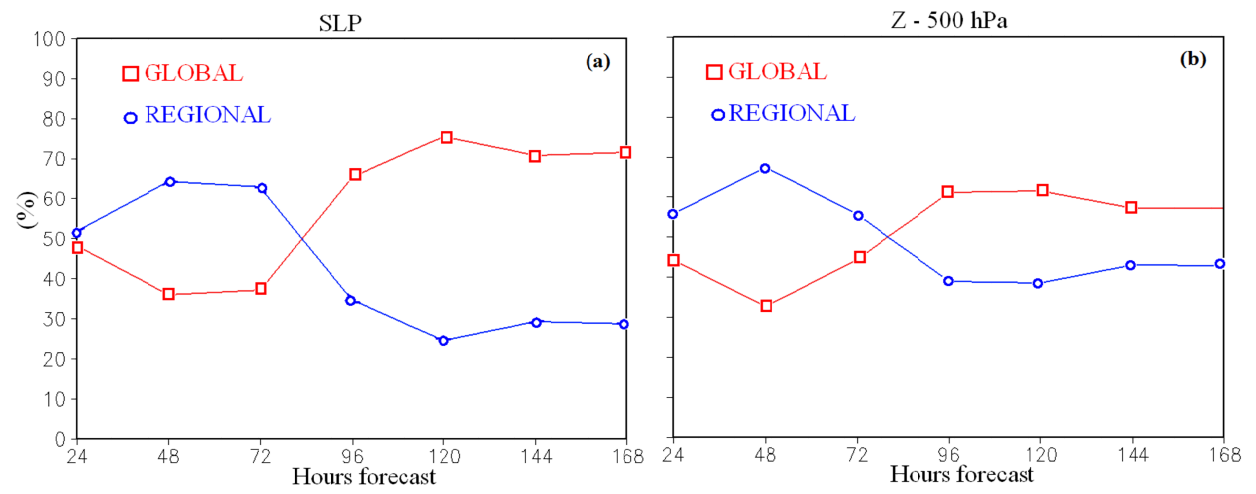

Figure 3. Evolution with time of the mean (over the whole domain) of the percentage of grid points in which the models had the smallest values of RMSE for winter and summer for (a) mean sea level pressure and (b) $500 \mathrm{hPa}$ geopotential.

RMSE in the first $72 \mathrm{~h}$ of integration; however for forecasts from $96 \mathrm{~h}$ on, the global models prevailed.

\subsection{SMEC}

In this section the means of some of the variables obtained by the SMEC system for summer and winter will be presented. In addition, a comparison between the predictions of the SMEC, the NCEP analyses (considered as "truth") and the Eta15 forecasts will be shown. The reason for introducing a comparison to only one of the models used in the system is simply to reduce the quantity of figures to be shown, and the choice of Eta15 model is due to the fact that this model usually provides smaller errors than the other models used, according the CPTEC/INPE model assessment system (http://avaliacaodemodelos.cptec.inpe.br/). Also in this section a statistical evaluation that includes the SMEC and all models used for producing it will be carried out. The RMSE is the primary statistical parameter used to assess forecast quality. For the surface variables (temperature at $2 \mathrm{~m}$, wind at $10 \mathrm{~m}$, mean sea level pressure and precipitation) observations at surface stations (METAR SYNOP and PCDs) will be used for verification, and due to the scarcity of observed data at higher levels the NCEP analysis will be used as the reference.

The mean for the summer trimester for some of the variables calculated from the NCEP analyses, and $168 \mathrm{~h}$ forecasts from the SMEC and Eta15 are shown in Fig. 4. The figure shows that the predictions produced by SMEC present patterns similar to those of the NCEP analyses, indicating that the SMEC provides consistent predictions. Comparison between the NCEP analyses and $168 \mathrm{~h}$ forecasts from SMEC versus Eta15 indicates that in general the SMEC forecasts are closer to the patterns seen in the NCEP analyses than the Eta15 forecasts. In the case of mean sea level pressure (Fig. 4a, b, c), the main differences are over the continent, where the Eta15 tends to underestimate the values. For air temperature at $850 \mathrm{hPa}$ (Fig. $4 \mathrm{~d}, \mathrm{e}, \mathrm{f}$ ) improvements of the SMEC over the Eta15 occur mainly on the east and west coasts of the continent, where the Eta15 indicates an average of $2 \mathrm{~K}$ less than the NCEP analysis. The SMEC can predict better than the Eta15 the configuration and magnitude of the mean geopotential field at $500 \mathrm{hPa}$ (Fig. 4g, h, i), especially at latitudes north of $30^{\circ} \mathrm{S}$. The average relative humidity at $850 \mathrm{hPa}$ from NCEP analyses (Fig. $4 \mathrm{j}$ ) shows a pattern typical of this season, i.e., high humidity in the region of convergence of the trade winds, the Amazon region, west-central region and the coastal strip of southern/southeastern Brazil. The driest region of the domain (less than $20 \%$ ) was over the Pacific Ocean, specifically at around $25^{\circ} \mathrm{S}$.

The $168 \mathrm{~h}$ forecasts from the SMEC (Fig. 4k) present a pattern consistent with that observed in the analysis, but the region with lower humidity is forecast to extend over a greater area, while on the northern coast of the continent and the region of the Intertropical Convergence Zone (ITCZ), SMEC overestimates the values of humidity. Like the SMEC, the Eta15 (Fig. 4l) can also identify the patterns of spatial distribution of relative humidity, however with a tendency to underestimate the humidity over the Atlantic at locations equatorward of southeastern Brazil and the central region of the continent. With respect to the meridional wind at $850 \mathrm{hPa}$ (Fig. $4 \mathrm{~m}, \mathrm{n}, \mathrm{o}$ ), the analysis indicates (Fig. $4 \mathrm{~m}$ ) the predominance of the southerly component of the meridional wind over the southern Pacific Ocean, except in the far northern portion of the domain. On the continent, the northerly component of the meridional wind prevails, the strongest lying along the Andes, characterized by the low-level jet. Over the Atlantic Ocean, more precisely between latitudes $25^{\circ}$ and $30^{\circ} \mathrm{S}$, a predominance of northerly winds during the summer can also be observed. The meridional component of the wind is observed to be from the south in the southern portion of the field, over the Atlantic Ocean and northeastern Brazil. The $168 \mathrm{~h}$ forecasts, both from the SMEC (Fig. $4 \mathrm{n}$ ) and the Eta15 (Fig. 4o), captured well the pattern of the meridional wind, but both tended to overestimate wind speed, with this feature more pronounced in the forecasts made by the Eta15. 


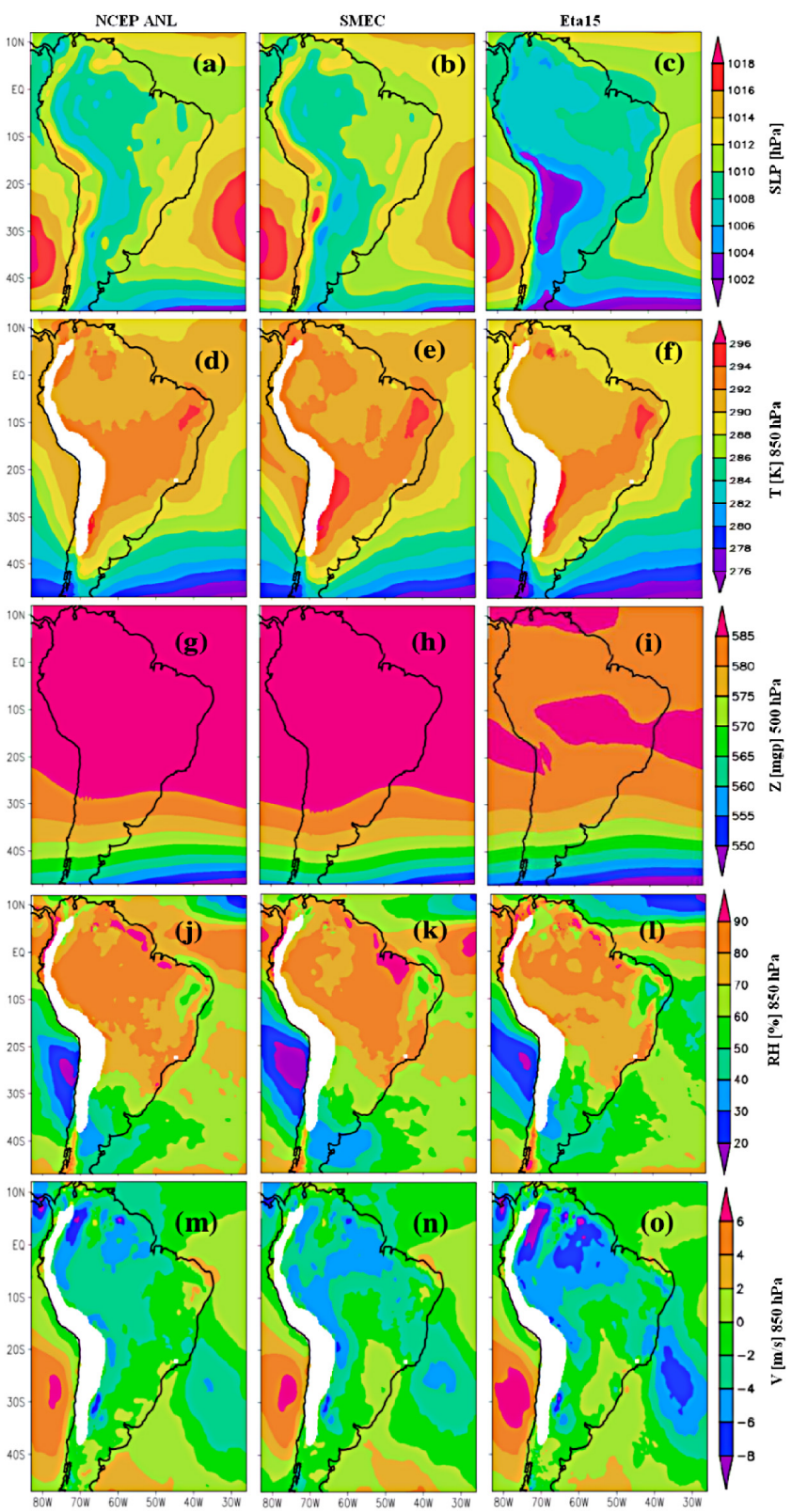

Figure 4. Mean for the summer trimester of the NCEP analyses (first column), and $168 \mathrm{~h}$ forecasts from the SMEC (second column) and Eta15 (third column) for mean sea level pressure (a, b, c), $850 \mathrm{hPa}$ temperature (d, e, f), $500 \mathrm{hPa}$ geopotential $(\mathbf{g}, \mathbf{h}, \mathbf{i})$, relative humidity $(\mathbf{j}, \mathbf{k}, \mathbf{l})$ and $850 \mathrm{hPa}$ meridional wind $(\mathbf{m}, \mathbf{n}, \mathbf{o})$.

The means for the winter trimester of the NCEP analyses and of the $168 \mathrm{~h}$ forecasts from the SMEC and Eta15 are shown in Fig. 5. Mean sea level pressure for winter, according to the NCEP analyses (Fig. 5a), indicates the predominance of a region of high pressure between latitudes $40^{\circ}$ and $15^{\circ} \mathrm{S}$, where the Atlantic and Pacific climatological highs are positioned, both having an average strength of around $1025 \mathrm{hPa}$. In the rest of the domain, the predominance
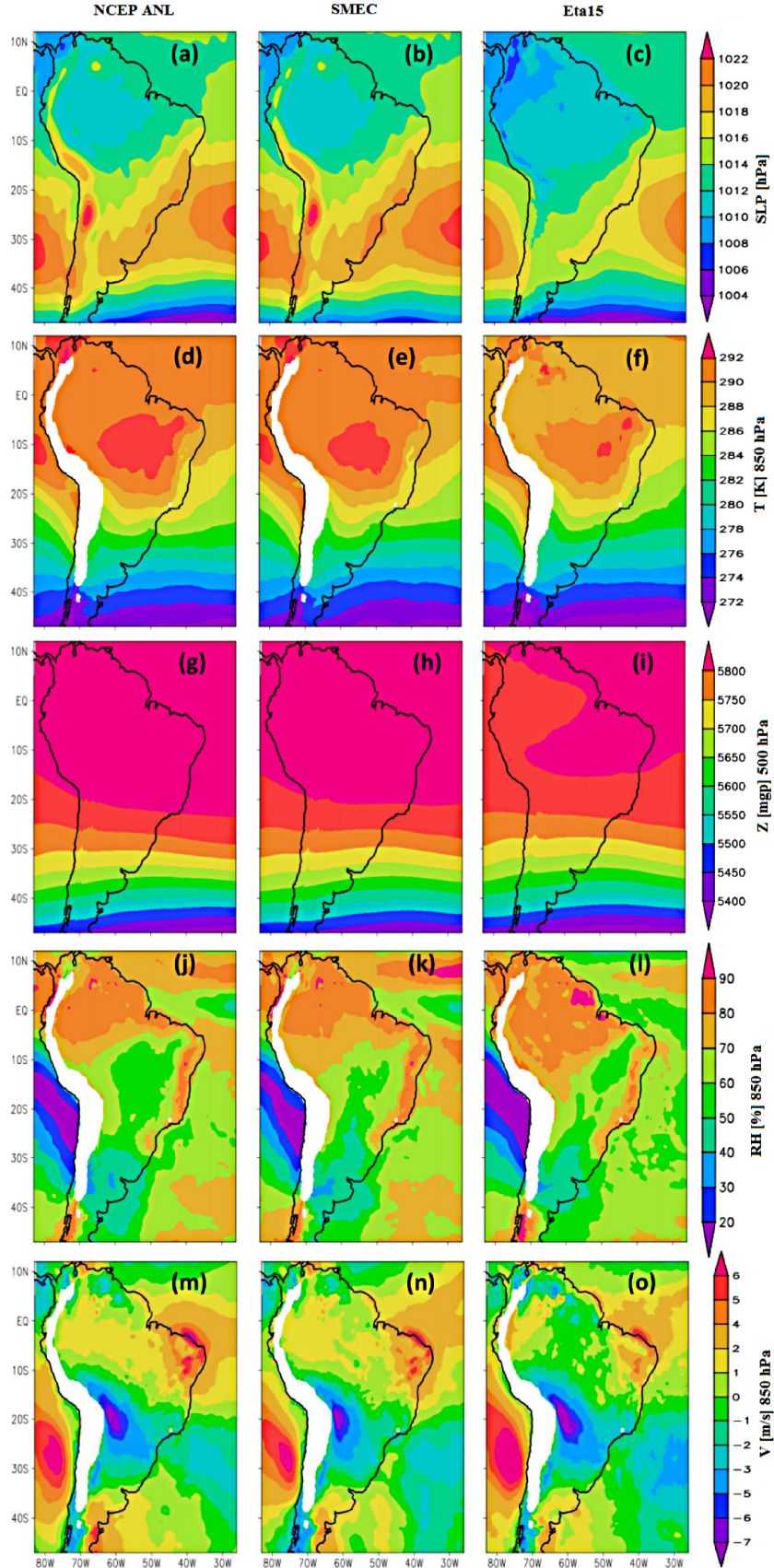

Figure 5. Means for the winter trimester of NCEP analyses (first column), and $168 \mathrm{~h}$ forecasts from SMEC (second column) and Eta15 (third column) for the variables mean sea level pressure (a, b, c), $850 \mathrm{hPa}$ temperature (d, e, f), $500 \mathrm{hPa}$ geopotential height $(\mathbf{g}, \mathbf{h}$, i), and $850 \mathrm{hPa}$ relative humidity $(\mathbf{j}, \mathbf{k}, \mathbf{l})$ and meridional wind $(\mathbf{m}$, n, o).

of regions with low pressure can be seen, with the most intense in the extreme south. The average for the same period of $168 \mathrm{~h}$ forecasts obtained from SMEC (Fig. 5b) shows patterns very similar to those seen in the NCEP analyses, both in location and in magnitude, except for the weakening of 

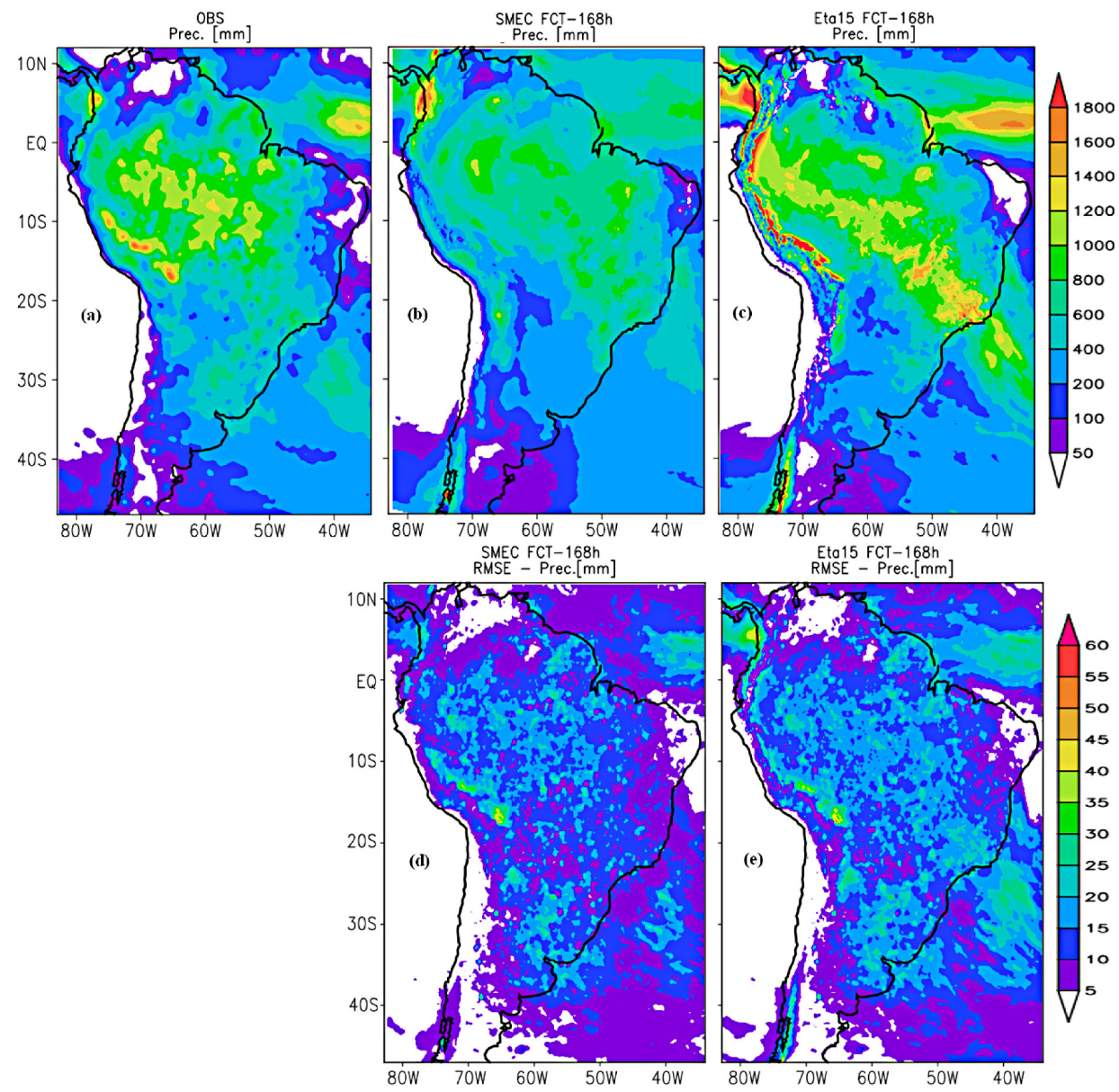

Figure 6. Precipitation accumulated during the summer period for MERGE (a), forecasts of $168 \mathrm{~h}$ from SMEC (b) and Eta15 (c), and their respective RMSE (d, e).

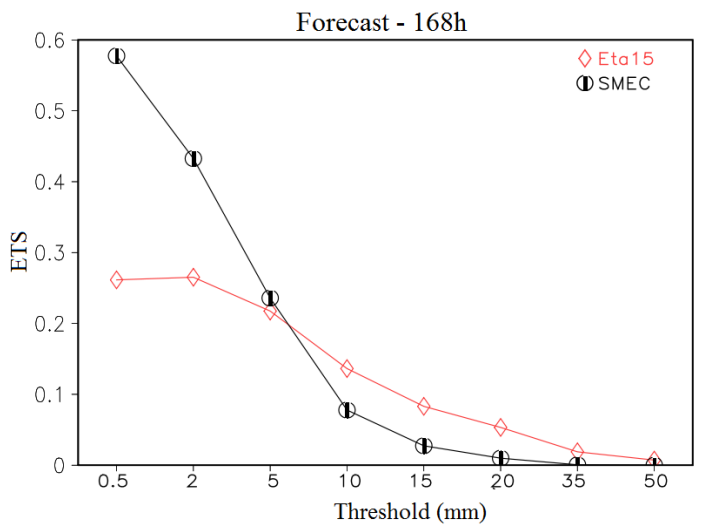

Figure 7. Equitable threat scores (ETSs) to $168 \mathrm{~h}$ forecast.

the low-pressure center south of the area. The average forecast for $168 \mathrm{~h}$ of Eta15 (Fig. 5c) shows similar patterns in the positioning of systems, but underestimates of the pressure values, i.e., shows weakening of highs and intensification of lows.
The mean air temperature at $850 \mathrm{hPa}$ from the NCEP analyses (Fig. 5d) shows a predominance of higher temperatures (291-294 K) mainly over the central region of South America. Comparison between this result and the $168 \mathrm{~h}$ forecast from SMEC (Fig. 5e) indicates very similar patterns throughout the integration domain, except for a narrow strip located on the northeast of the domain, where the average temperature was slightly underestimated. The average of the Eta15 predictions for the latitudes below $20^{\circ} \mathrm{S}$ (Fig. 5f) is also consistent (position and intensity) with the analyses; however, in regions where the highest temperature values occurred, the Eta15 forecasts showed a trend toward underestimation.

As was seen for the summer period, the average of the geopotential field at $500 \mathrm{hPa}$ forecasted by the SMEC (Fig. 5h) is quite similar to the average of the NCEP analyses (Fig. 5i), both in terms of intensity and in terms of configuration. The Eta15 can capture the values of geopotential reasonably well in the southern portion of the domain, yet north of $40^{\circ} \mathrm{S}$ it tends to underestimate them. In the case of relative humidity (Fig. 5j, k, l) it is observed that, in general, the SMEC (Fig. 5k) captures very well the distribution 

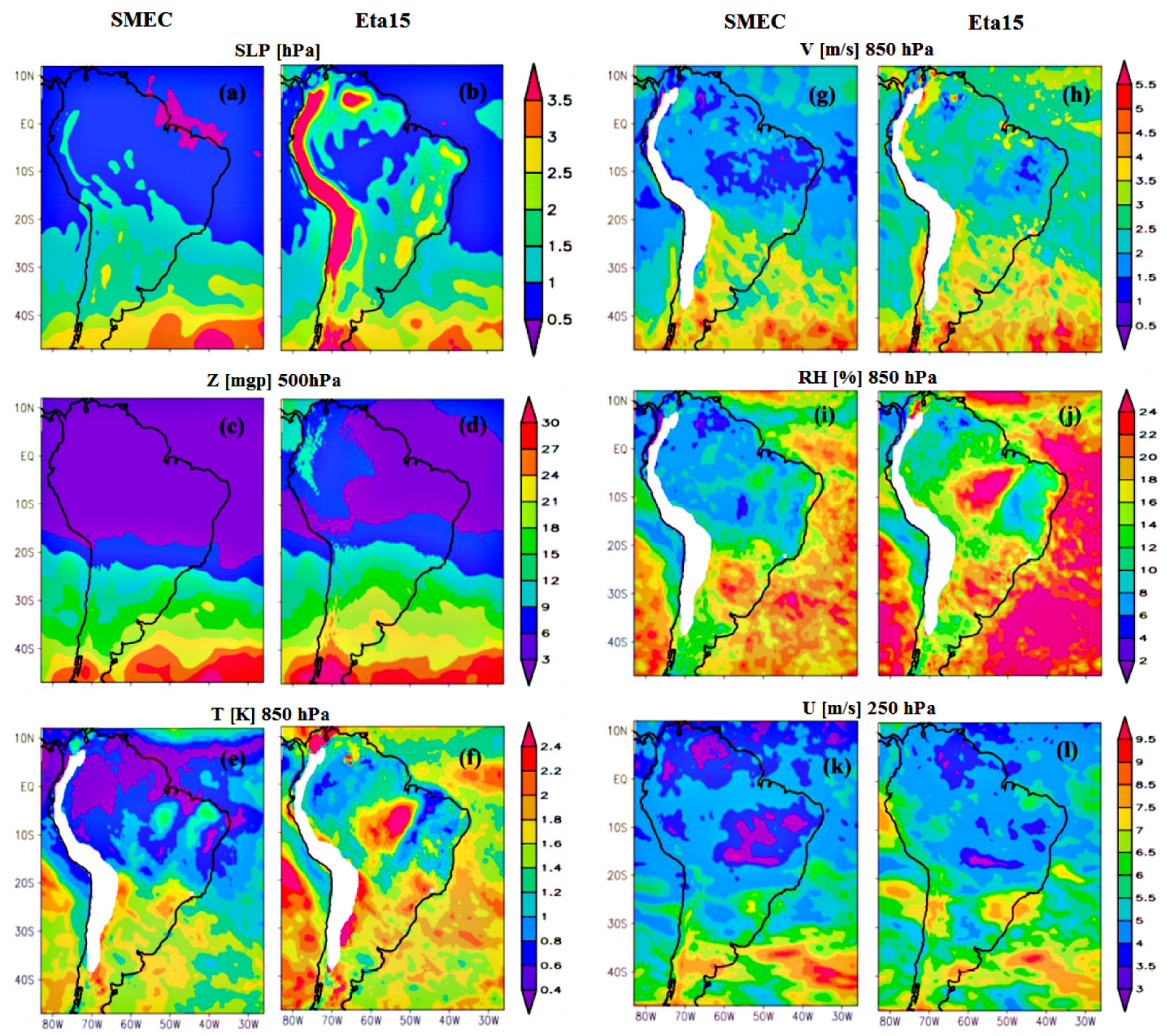

Figure 8. Spatial distribution of the RMSE at $48 \mathrm{~h}$ of integration for the winter period for SMEC (left column) and Eta15 (right column), for mean sea level pressure (a, b), $500 \mathrm{hPa}$ geopotential height $(\mathbf{c}, \mathbf{d}), 850 \mathrm{hPa}$ air temperature $(\mathbf{e}, \mathbf{f}), 850 \mathrm{hPa}$ meridional wind $(\mathbf{g}, \mathbf{h}), 850 \mathrm{hPa}$ relative humidity $(\mathbf{i}, \mathbf{j})$ and $250 \mathrm{hPa}$ zonal wind $(\mathbf{k}, \mathbf{l})$.

of moisture; however the model overestimates it in the northeast, while Eta15 (Fig. 5j) overestimates it in the northern part of the continent. With respect to the meridional wind at $850 \mathrm{hPa}$ (Fig. 5m, n, o), both the SMEC (Fig. 5) and the Eta15 (Fig. 5o) can provide good average position of the most intense cores; however, the magnitude of the meridional wind is better predicted by SMEC, especially over the Amazon region northeast of Brazil.

Figure 6a shows total rainfall accumulated during the summer period. The rainfall was obtained from the MERGE precipitation product (Rozante et al., 2010) available at CPTEC/INPE (ftp://ftp1.cptec.inpe.br/modelos/io/produtos/ MERGE).

During the summer, rainfall was concentrated over the Amazon region, southern, southeastern and west central Brazil, Peru, Bolivia and the equatorial and subtropical Atlantic. The $168 \mathrm{~h}$ SMEC forecasts (Fig. 6b) generally manage to capture the patterns of the precipitation regime, but show tendencies to underestimate the intensity of rainfall as well as to extend the area of precipitation, mainly in the northeastern region of Brazil. Like the SMEC, the Eta15 (Fig. 6c) had also captured the pattern of spatial distribution of rainfall, however with a tendency to overestimate it, especially in the regions near the Andes mountains, and the west-central and southeastern regions of Brazil.

Comparison between the RMSE of summer-period precipitation at $168 \mathrm{~h}$ of integration, between the SMEC (Fig. 6d) and Eta15 (Fig. 6e), shows a reduction of errors by the SMEC over practically the whole domain. Similar results (not shown) were also observed for other times of integration.

Although the results for the RMSE have shown a considerable reduction in the magnitude of the errors, the quantitative analysis presented by the equitable threat score (ETS) (Mesinger, 2008) indicates that the SMEC performed better only for precipitation thresholds up to $5 \mathrm{~mm}$ (light rain); for the thresholds for moderate to heavy rain (above $10 \mathrm{~mm}$ ), the performance of the SMEC was inferior to that of the Eta15. 

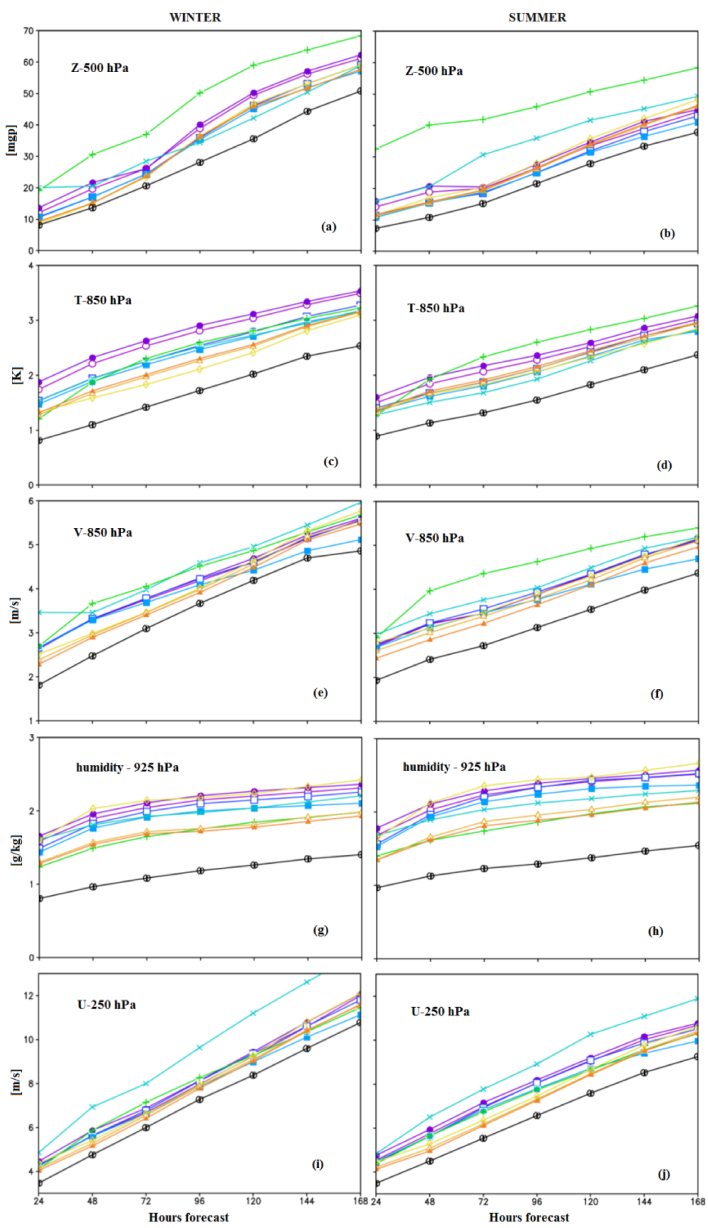

Figure 9. Time evolution of the spatial mean of the RMSE (calculated from the NCEP analyses) of $500 \mathrm{hPa}$ geopotential height (a, b), $850 \mathrm{hPa}$ air temperature (c, d), $850 \mathrm{hPa}$ meridional wind (e, f), $925 \mathrm{hPa}$ specific humidity $(\mathbf{g}, \mathbf{h})$ and $250 \mathrm{hPa}$ zonal wind $(\mathbf{i}, \mathbf{j})$ for the winter (left column) and summer (right column) trimesters.

Figure 7 illustrates this behavior at $168 \mathrm{~h}$ of integration. The falloff in the SMEC's performance for high thresholds is due to the weighting method; since precipitation is a spatially discrete variable, errors in the positioning of the precipitation cores make them appear weaker and more spread out.

The spatial distributions of the RMSE (calculated with respect to the NCEP analyses) for $48 \mathrm{~h}$ forecasts for the winter period from SMEC and Eta15 are shown in Fig. 8. In the case of mean sea level pressure (Fig. 8a, b), errors are lower for SMEC than Eta15 in almost any field, except in the southern portion of the ocean. The reduction of errors by the SMEC is quite evident, primarily on the mainland (the Andes, Venezuela, southern Argentina and northeastern Brazil). The geopotential height at $500 \mathrm{hPa}$ (Fig. 8c, d) also indicates smaller RMSE values for the SMEC over most of the domain except over the southern Atlantic Ocean. The analyses for the variables air temperature, meridional wind and relative humidity (Fig. 8e, f, g, h, i, j), all at $850 \mathrm{hPa}$, show much
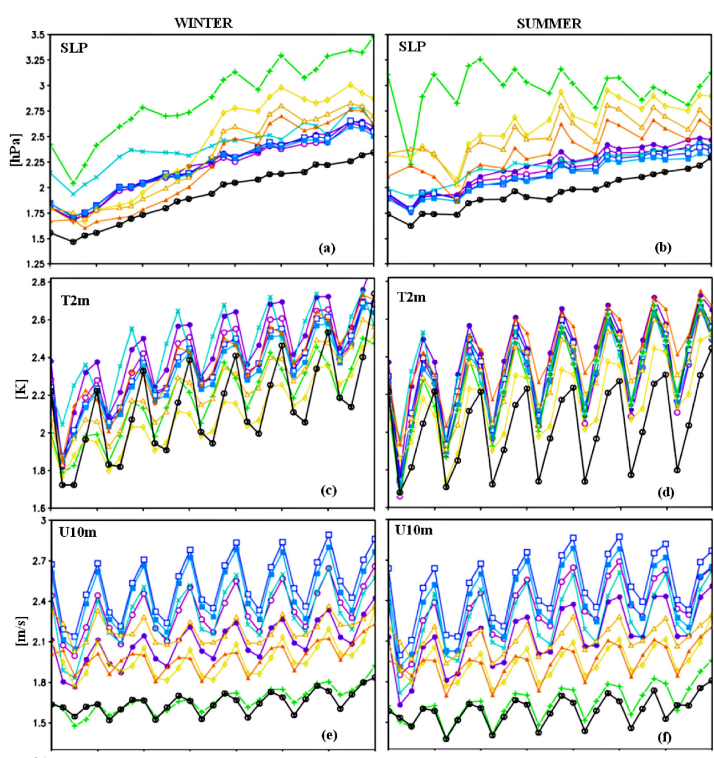

+ BRAMS 20

Eta:

$\triangle$ Eta40ZHAO

$\oplus \mathrm{SMEC}$

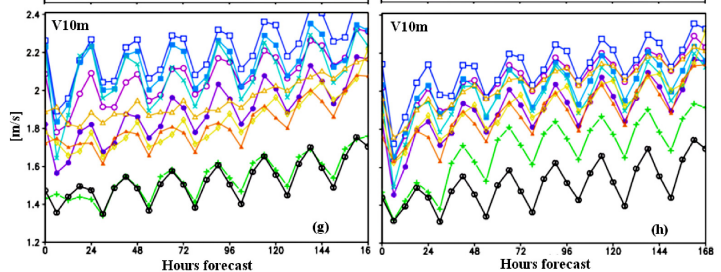

Figure 10. Time evolution of the spatial mean of the RMSE (calculated with respect to observations) of mean sea level pressure (a, b), temperature at $2 \mathrm{~m} \mathrm{(c,} \mathrm{d),} \mathrm{zonal} \mathrm{wind} \mathrm{at} 10 \mathrm{~m}(\mathbf{e}, \mathbf{f})$, meridional wind at $10 \mathrm{~m} \mathrm{(g,} \mathrm{h)} \mathrm{for} \mathrm{the} \mathrm{winter} \mathrm{(left} \mathrm{column)} \mathrm{and} \mathrm{summer} \mathrm{(right}$ column) trimesters.

lower magnitudes of errors in fields related to SMEC; only in a few small regions does Eta15 present smaller errors. The analysis for the zonal wind at $250 \mathrm{hPa}$ (Fig. 8k, l) indicates that, in by far the largest portion of the area north of latitude $30^{\circ} \mathrm{S}$, errors are smaller in the SMEC than in the Eta15; however, in latitudes south of $30^{\circ} \mathrm{S}$ errors of the SMEC forecasts are larger, especially in the region near the subtropical jet. Analyses carried out for summer and for other lengths of integration (not shown) also showed better quality in the SMEC forecasts as compared with the Eta15.

Figure 9 shows the temporal evolution of the spatial average RMSE for the winter and summer trimesters, where the NCEP analyses were used as "truth". Through the analysis of this figure, we can affirm that the magnitudes of errors for the winter quarter are generally higher than those in summer. This fact is due to the larger number of systems that cross the continent during the winter. In general, the errors in SMEC were relatively smaller than those in all models used for its calculation and at all times of integration.

The calculation of the RMSE for near-surface variables was performed using observational data from weather stations from METAR and SYNOP and automatic data collection platforms (DCPs). Figure 10 shows the time evolution of 
the RMSE for the winter and summer trimesters, including the mean of all existing observations from METAR, SYNOP and PCDs in the domain. Details of this evaluation metric can be found in Moreira et al. (2013). In general, just as was observed in the errors obtained by use of the NCEP analyses, the magnitudes of the RMSE for the winter trimester are higher than for summer. In the case of mean sea level pressure (Fig. 10a, b) it is observed that, throughout the integration, the SMEC produced errors smaller than those of all the other models considered. The results for the temperatures at $2 \mathrm{~m}$ (Fig. 10c, d) indicate that around the time of minimum temperature SMEC behaves better than all other models. However, in winter the maximum temperatures obtained by SMEC had higher errors than the regional models. It can be seen that all global models showed very significant errors for forecasts of maximum temperature. Although the weight of these models was lower in calculating the SMEC, the contribution of all global models caused the SMEC to forecast a maximum temperature that was not very accurate during the winter. For the zonal wind at $10 \mathrm{~m}$ (Fig. 10e, f), the SMEC proved far more accurate than the other models, except BRAMS20, which showed errors of the same order of magnitude as SMEC. In the case of the meridional wind at $10 \mathrm{~m}$ (Fig. $10 \mathrm{~g}, \mathrm{~h}$ ) the SMEC and the BRAMS20 also stood out, although in the summer (Fig. 10h) results for the BRAMS20 beyond $24 \mathrm{~h}$ of integration began to diverge considerably, while the SMEC continued with errors smaller than those of the other models.

\section{Conclusions}

In this paper a method was proposed (the SMEC) for improving the quality of numerical weather forecasts, using an expanded set of forecasts from various numerical models. This method was applied to nine models run operationally at CPTEC/INPE with domains over South America. Results were analyzed for the summer and winter trimesters, using observed data and NCEP analyses, and compared with the model with the best performance of any in the group (Eta15).

The analysis with respect to the contributions of each of the SMEC's component models showed that regional models make their greatest contributions in the first $72 \mathrm{~h}$ of integration; beyond this time the forecasts by global models are more accurate. This fact makes it clear that a combination of the predictions of all the models in question can produce a product that will combine what is best in each model.

The subjective assessment of the Eta 15 and SMEC predictions, considering the NCEP analyses as "truth", showed that the forecasts generated from the SMEC behaved more like the NCEP analyses than did the Eta15 forecasts, in all situations evaluated. The results with respect to RMSE, calculated relative to the NCEP analyses and the observed data, showed that forecasts prepared by SMEC system produced errors of smaller magnitude than those of any of the models used, for practically all variables, all levels and for all durations of integration evaluated.

For precipitation, it can be seen that the SMEC succeeded in capturing the patterns of precipitation, but it shows a tendency to underestimate intense rainfall and increase the area of occurrence of light rainfall. This behavior is associated with the weighting method applied in the calculation of the SMEC. In this regard it is intended, in future work, to apply special methods for obtaining the precipitation field, such as using the ETS itself in the determination of weights.

Based on the results presented we can conclude that the method proposed in this paper can considerably improve the results provided by numerical models and can be extremely important for studies of atmospheric phenomena, as well as assisting with the preparation of forecasts. In this sense, the SMEC product shows promising potential for operational applications in any weather forecasting center.

The product has been processing since January 2014, the files for the past five days can be download at $\mathrm{ftp} / / / \mathrm{ftp} 1 . c p t e c . i n p e . b r / m o d e l o s /$ io/produtos/SMEC.

Acknowledgements. We acknowledge $\mathrm{CNPq}$ for financial assistance through the project "Predictability of extreme precipitation events by means of an expanded set of numerical predictions" (project number: 479638/2011-0), and the Center for Weather Forecasting and Climate Studies, National Institute for Space Research (CPTEC/INPE) for making its infrastructure available.

Edited by: J. Koller

\section{References}

Chakraborty, A., Krishnamurti, T. N., and Gnanaseelan, C.: Prediction of the diurnal cycle using a multimodel superensemble. Part II: Clouds, Mon. Weather Rev., 135, 4097-4116, 2007.

Chandler, R. E.: Exploiting strength, discounting weakness: combining information from multiple climate simulators, Phil. Trans. R. Soc. A, 371, 20120388, doi:10.1098/rsta.2012.0388, 2013.

Christensen, J. H., Kjellström, E., Giorgi, F., Lenderink, G., and Rummukainen, M.: Weight assignment in regional climate models, 44, 179-194, doi:10.3354/cr00916, 2010.

Cintineo, R. M. and Stensrud, D. J.: On the Predictability of Supercell Thunderstorm Evolution, J. Atmos. Sci., 70, 1993-2011, doi:10.1175/JAS-D-12-0166.1, 2013.

Déqué, M. and Somot, S.: Weighted frequency distributions express modelling uncertainties in the ENSEMBLES regional climate experiments, Clim. Res., 44, 195-209, doi:10.3354/cr00866, 2010.

Johnson, C. and Swinbank, R.: Medium-range multimodel ensemble combination and calibration, Q. J. Roy. Meteorol. Soc., 135, 777-794, 2009.

Knutti, R., Furrer, R., Tebaldi, C., Cermak, J., and Meehl, G. A.: Challenges in combining projections from multiple climate models, J. Climate, 23, 2739-2758, doi:10.1175/2009JCLI3361.1, 2010. 
Kotal, S. D. and Roy Bhowmik, S. K.: A Multimodel Ensemble (MME) Technique for Cyclone Track Prediction over the North Indian Sea, Geofizika, 28 275-291, 2011.

Krishnamurti, T. N., Kishtawal, C. M., LaRow, T. E., Bachiochi, D. R., Zhang, Z., Williford, C. E., Gadgil, S., and Surendran, S.: Improved weather and seasonal climate forecasts from multimodel superensemble, Science, 285, 1548-1550, 1999.

Krishnamurti, T. N., Kishtawal, C. M., Zhang, Z., LaRow, T., Bachiochi, D., Williford, E., Gadgil, S., and Surendran, S.: Multimodel Ensemble Forecasts for Weather and Seasonal Climate, J. Climate, 13, 4196-4216, doi:10.1175/15200442(2000)013<4196:MEFFWA>2.0.CO;2, 2000a.

Krishnamurti, T. N., Kishtawal, C. M., Zhang, Z., LaRow, T. E., Bachiochi, D. R., Williford, C. E., Gadgil, S., and Surendran, S.: Improving tropical precipitation forecasts from a multianalysis superensemble, J. Climate, 13, 4217-4227, 2000b.

Krishnamurti, T. N., Sanjay, J., Mitra, A. K., and Vijaya Kumar, T. S. V.: Determination of Forecast Errors Arising from Different Components of Model Physics and Dynamics, Mon. Weather Rev., 132, 2570-2594, doi:10.1175/MWR2785.1, 2004.

Krishnamurti, T. N., Mishra, A. K., Chakraborty, A., and Rajeevan, M.: Improving Global Model Precipitation Forecasts over India Using Downscaling and the FSU Superensemble, Part I: 1-5-Day Forecasts, Mon. Weather Rev., 137, 2713-2735, 2009.

Kumar, A., Mitra, A. K., Bohra, A. K., Iyengar, G. R., and Durai, V. R.: Multi-model ensemble (MME) prediction of rainfall using neural networks during monsoon season in India, Meteorol. Appl., 19, 161-169, 2012.

Lenartz, F., Mourre, B., Barth, A., Beckers, J.-M., Vandenbulcke, L., and Rixen, M.: Enhanced ocean temperature forecast skills through 3-D super-ensemble multi-model fusion, Geophys. Res. Lett., 37, L19606, doi:10.1029/2010GL044591, 2010.

Lorenz, E. N.: A study of the predictability of a 28 -variable atmospheric model, Tellus, 17, 321-333, 1965.

Lorenz, E. N.: The predictability of a flow which possesses many scales of motion, Tellus, 21, 289-307, 1969.

Mesinger, F.: Bias Adjusted Precipitation Threat Scores, Adv. Geosci., 16, 137-142, doi:10.5194/adgeo-16-137-2008, 2008.

Mitra, A. K., Iyengar, G. R., Durai, V. R., Sanjay, J., Krishnamurti, T. N., Mishra, A., and Sikka, D. R.: Experimental Real-Time Multi-Model Ensemble (MME) Prediction of Rainfall During Monsoon 2008: Large-Scale Medium-Range Aspects, J. Earth Syst. Sci., 120, 27-52, 2011.
Ngan, K. and Eperon, G. E.: Middle atmosphere predictability in a numerical weather prediction model: revisiting the inverse error cascade, Q. J. Roy. Meteorol. Soc., 138, 1366-1378, 2012.

Roy Bhowmik, S. K. and Durai, V. R.: Multimodel ensemble forecasting of rainfall over Indian monsoon region, Atmosfera, 21, 225-239, 2008.

Roy Bhowmik, S. K. and Durai, V. R.: Application of multi-model ensemble techniques for real time district level rainfall forecasts in short range time scale over Indian region, Meteorol. Atmos. Phys., 106, 19-35, 2010.

Rozante, J. R, Moreira, D. S., de Goncalves, L. G. G., and Vila, D. A.: Combining TRMM and Surface Observations of Precipitation: Technique and Validation over South America, Weather Forecast., 25, 885-894, doi:10.1175/2010WAF2222325.1, 2010.

Saulo, A. C., Seluchi, M., Campetella, C., and Ferreira, L.: Error evaluation of NCEP and LAHM regional model daily forecasts over southern South America, Weather Forecast., 16, 697-712, 2001.

Silva Dias, P. L., Moreira, D. S., and Neto, G. D.: The MASTER Model Ensemble System (MSMES). Preprints, Eighth Int. Conf. on Southern Hemisphere Meteorology and Oceanography, Foz do Iguaçu, Brazil, Amer. Meteor. Soc., 4 pp., 2006.

Tebaldi, C., Mearns, L. O., Nychka, D., and Smith, R. L.: Regional probabilities of precipitation change: A bayesian analysis of multimodel simulations, Geophys. Res. Lett., 31, L24213, doi:10.1029/2004GL021276, 2004.

Thompson, P.: Uncertainty of initial state as a factor in predictability of large-scale atmospheric flow patterns, Tellus, 9, 275-295, 1957.

van Lier, W. M., Vukicevic, T., and Posselt, D. J.: Quantification of Cloud Microphysical Parameterization Uncertainty Using Radar Reflectivity, Mon. Weather Rev., 140, 3442-3466, 2012.

Weigel, A. P., Knutti, R., Liniger, M. A., and Appenzeller, C.: Risks of model weighting in multimodel climate projections, J. Climate, 23, 4175-4191, doi:10.1175/2010JCLI3594.1, 2010.

Yun, W.-T., Stefanova, L., and Krishnamurti, T. N.: Improvement of the multimodel superensemble technique for seasonal forecasts, J. Climate, 16, 3834-3840, 2003.

Zhu, H. and Thorpe, A.: The predictability of extra-tropical cyclones: the influence of the initial condition and model uncertainties, J. Atmos. Sci., 63, 1483-1497, 2006. 